\title{
Aerobic Bacterial Profile and Antibiotic Susceptibility Pattern of Chronic Suppurative Otitis Media in a Tertiary Care Centre in Kerala
}

\author{
Aishwarya B. ${ }^{1}$, Prithi Nair K. ${ }^{2}$ \\ ${ }^{1}$ Senior Resident, Department of Microbiology, Government Medical College, Thrissur, Kerala, India. ${ }^{2}$ Professor and \\ HOD, Department of Microbiology, Government Medical College, Thrissur, Kerala, India.
}

\section{ABSTRACT}

\section{BACKGROUND}

Chronic suppurative otitis media is a notorious middle ear infection in the developing countries which if left untreated can lead to serious intra and extracranial complications. We wanted to identify the bacterial pathogens causing CSOM and to determine their antibiotic susceptibility pattern.

\section{METHODS}

The in-patients \& out-patients from the Department of E.N.T. over a period of one year were recruited and evaluated in the study. Ear swabs collected from the 135 clinically diagnosed cases of CSOM were processed in the microbiology laboratory. The bacterial isolates were identified, and their antibiotic susceptibility testing was done.

\section{RESULTS}

Bacterial growth was obtained from 100 out of the 135 samples of which $37.5 \%$ of the isolates were Pseudomonas aeruginosa, 21.5\% Staphylococcus aureus, 5.9\% Klebsiella pneumoniae, 3.7\% Escherichia coli etc. Maximum antibiotic resistance was observed towards Ciprofloxacin, Gentamicin and Ceftazidime in Pseudomonas aeruginosa. Among the multi-drug resistant Gram-negative isolates, 20 were Extended Spectrum Beta Lactamase producers, 10 were AmpC beta lactamase producers and 10 were Metallo Beta Lactamase producers.

\section{CONCLUSIONS}

The bacterial isolates were found to be highly resistant to fluroquinolones and aminoglycosides, the most commonly used topical antibiotics for CSOM, in this study. Culture and antibiotic susceptibility testing of the bacterial isolates are therefore essential to help clinicians in better management of the cases and reduce the emergence of antibiotic resistant strains.

\section{KEY WORDS}

CSOM, Antibiotic Susceptibility, Pseudomonas aeruginosa, Staphylococcus aureus
Corresponding Author:

Dr. Aishwarya B.,

Chinmaya NH by Pass Road,

Nadathara P. O., Thrissur-680751,

Kerala, India.

E-mail: aishwaryabkk@gmail.com

DOI: $10.14260 / j e m d s / 2019 / 589$

Financial or Other Competing Interests: None.

How to Cite This Article:

Aishwarya B, Nair PK. Aerobic bacterial profile and antibiotic susceptibility pattern of chronic suppurative otitis media in a tertiary care centre in Kerala. J. Evolution Med. Dent. Sci. 2019;8(35):2713-2718, DOI: $10.14260 / j e m d s / 2019 / 589$

Submission 08-07-2019,

Peer Review 15-08-2019,

Acceptance 21-08-2019,

Published 02-09-2019. 


\section{BACKGROUND}

Chronic suppurative otitis media is characterized by intermittent or persistent purulent discharge through the perforated tympanic membrane. ${ }^{1}$ It is a disease of multiple etiologies and is known for its persistence and recurrence despite treatment. Infection can spread from middle ear to adjacent vital structures like the mastoid, facial nerve, labyrinth, lateral sinus, meninges and brain resulting in mastoid abscess, facial nerve paralysis, deafness, lateral sinus thrombosis, meningitis and intracranial abscess. Of all the complications, preventable hearing loss is nearly always significant particularly in the developing countries. The incidence of CSOM is at a rise in the developing countries as a result of poor hygienic practices and lack of health education. The study of bacterial profile and drug susceptibility is essential to help the treating physician plan the general management of CSOM and it is necessary for the ENT surgeon to make the discharging ear dry by myringoplasty and ossiculoplasty. However, the antibiogram of these organisms causing CSOM has been found to vary with time and geographical area as well as from continent to continent mostly because of the indiscriminate use of the antibiotics. The most commonly obtained bacterial isolate from the ear discharge of a patient with CSOM is Pseudomonas aeruginosa. The other bacterial isolates include Staphylococci, Escherichia coli, Klebsiella pneumoniae and Streptococcus species. Detection of ESBL, MBL \& Amp C enzymes are difficult, as their presence does not always produce a resistant phenotype on conventional disc diffusion or automated susceptibility testing methods. These enzymes are often associated with potentially fatal laboratory reports of false susceptibility to $\beta$-lactams phenotypically. 2 Thus, their accurate detection and characterization is important from epidemiological, clinical, laboratory and infection control point of view.

\section{METHODS}

This cross-sectional study was done in a tertiary care setting. In patients \& Outpatients from the Department of E.N.T. were recruited and the evaluation was done in the Department of Microbiology, Government Medical College, Thrissur. The study was conducted over a period of one year. Sample size was calculated to be 135 (Calculated from former similar study with formula $4 \mathrm{pq} / \mathrm{d} 2$ where $\mathrm{p}$ is the prevalence of Gram-Positive organism (42.62\%), ${ }^{3} \mathrm{q}$ is 100 -p and $\mathrm{d}$ is $20 \%$ of p). Patients who were suffering from CSOM diagnosed after thorough clinical evaluation by ENT Surgeon and who were willing to participate in the study were included in the study. Patients below 5 years were excluded, in view of difficulty in obtaining proper sample. Patient's with history of ear discharge of less than 3-month duration were also excluded. Before collecting the ear discharge, the external auditory canal was cleared of cerumen, using normal saline and allowed to dry. The middle ear discharge was then collected aseptically by the otorhinolaryngologist from the tympanic cavity with two thin, sterile cotton swabs. These swabs were labeled and then transported immediately to the microbiology laboratory for further processing.

A thin smear was made on a clean glass slide which was fixed by heating. Gram staining was done for the smears and was examined under oil immersion objective of the light microscope to note the presence or absence of inflammatory cells, Gram reaction, morphology and arrangement of the bacteria. The swabs were then inoculated on Blood agar (BA), MacConkey agar (MA), and Tellurite blood Agar (TA) and incubated at $37^{\circ} \mathrm{C}$ overnight. If there was no growth, the plates were further incubated for 48 hours and then reported as no growth. If growth was seen then the colony morphology was studied on BA, MA and TA plates after which the bacterial species were identified based on the standard biochemical reactions. Antibiotic susceptibility testing for each organism was done according to the Kirby-Bauer disc diffusion method on Muller Hinton Agar as per Clinical and Laboratory Standards Institute (CLSI) guidelines. ${ }^{4}$ Extended Spectrum Beta Lactamases, Metallo Beta Lactamases \& Amp C Beta lactamases were tested for in the resistant strains of Enterobacteriaceae. The data were analysed using descriptive statistical tools like percentages and frequency distributions. SPSS software version 20 was used for this purpose.

\section{RESULTS}

135 samples collected over a period of one year were studied. $37 \%$ of the isolates obtained were Pseudomonas aeruginosa, $21.5 \%$ were Staphylococcus aureus. Klebsiella pneumoniae contributed to 5.9\%, Escherichia coli 3.7\%, Enterococcus faecalis 1.5\%, Enterobacter aerogenes 1.5\%, Streptococcus pyogenes and Proteus mirabilis $0.7 \%$. Pseudomonas aeruginosa was highly susceptible to Piperacillin-tazobactam (88\%) and Imipenem (86\%). But only few isolates were susceptible to Ciprofloxacin (36\%) and Gentamicin (48\%). Similarly, among Enterobacteriaceae, $50 \%$ of Klebsiella pneumoniae, 50\% Enterobacter aerogenes, 20\% Escherichia coli were susceptible to Ciprofloxacin. Out of the 29 isolates of Staphylococcus aureus, all were susceptible to Cefoxitin (Surrogate marker for mecA mediated oxacillin resistance). 20 isolates were found to be positive for ESBL which consisted of Pseudomonas aeruginosa, Klebsiella pneumoniae, Escherichia coli and Proteus mirabilis. 10 isolates each were positive for AmpC and MBL production.

\begin{tabular}{|c|c|c|}
\hline Age Group of Patients & Frequency & Percent \\
\hline 15 \& Below & 21 & 15.6 \\
\hline $16-25$ & 23 & 17 \\
\hline $26-35$ & 19 & 41.1 \\
\hline $36-45$ & 28 & 20.7 \\
\hline $45-55$ & 25 & 18.5 \\
\hline $56-65$ & 12 & 8.9 \\
\hline Above 65 & 7 & 5.2 \\
\hline \multicolumn{3}{|c|}{ Age Group of Patients } \\
\hline
\end{tabular}



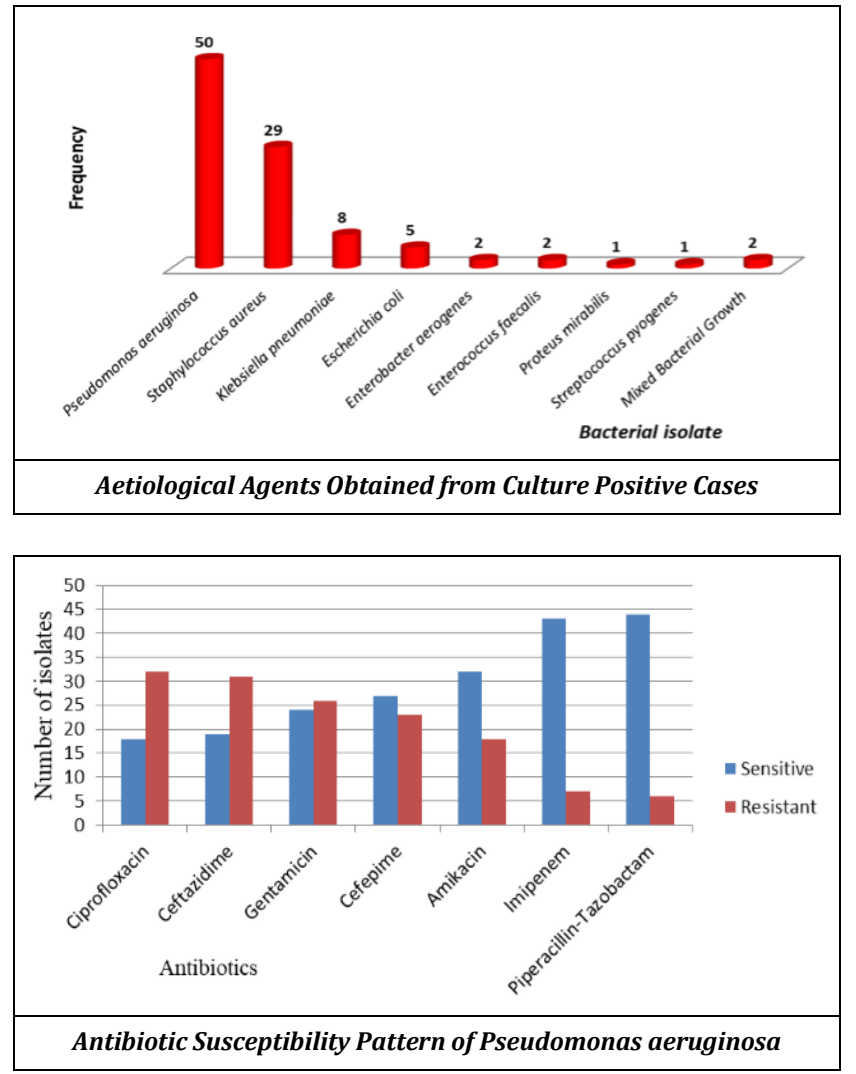

\section{Antibiotic Susceptibility Testing of Pseudomonas aeruginosa}

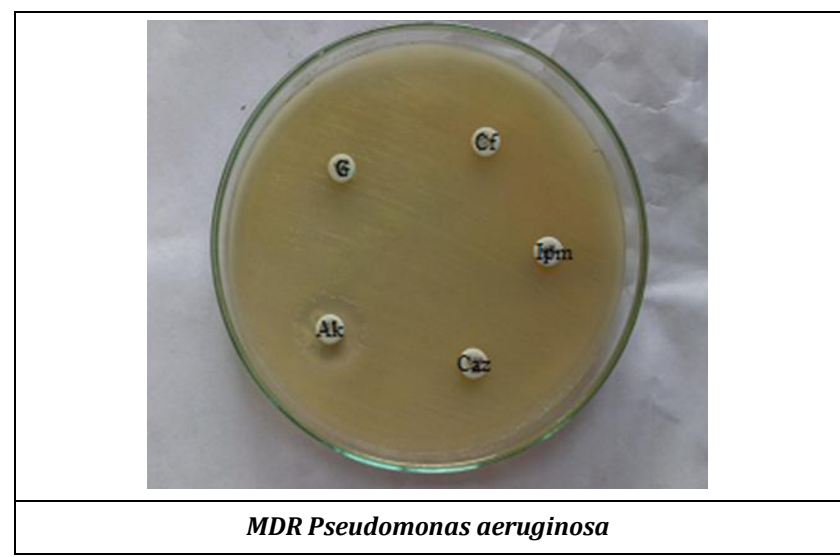

Ak- Amikacin, Cf- Ciprofloxacin, Caz- Ceftazidime, CpmCefepime, G- Gentamicin, Ipm- Imipenem

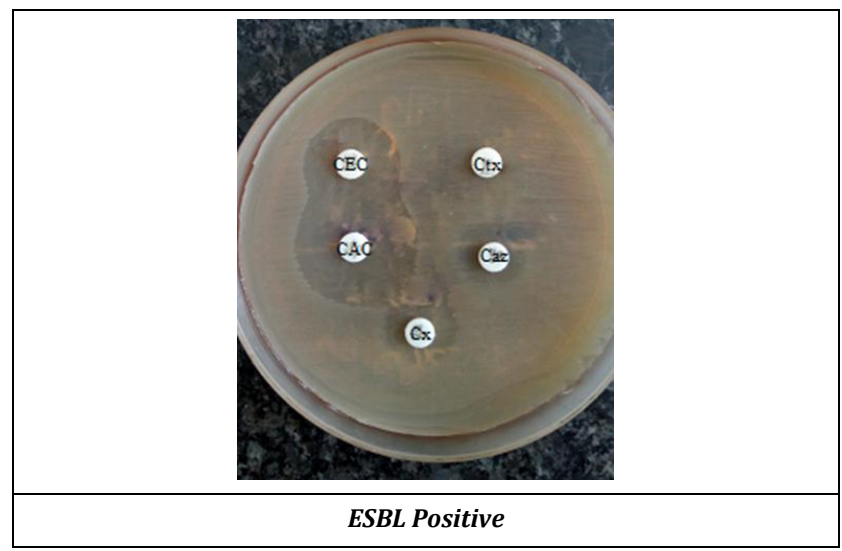

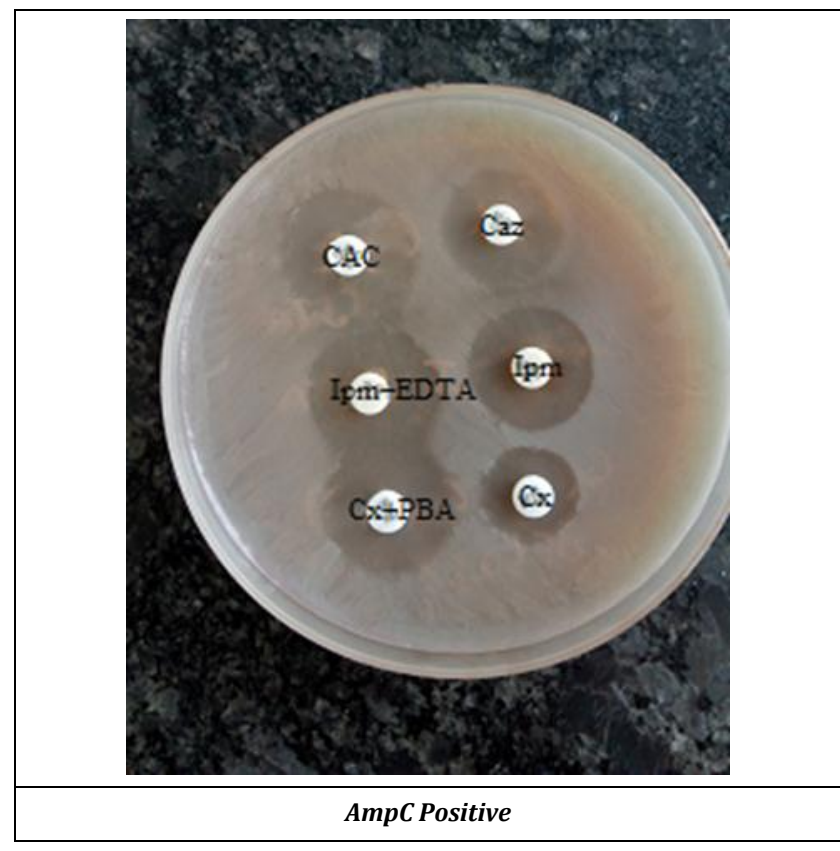

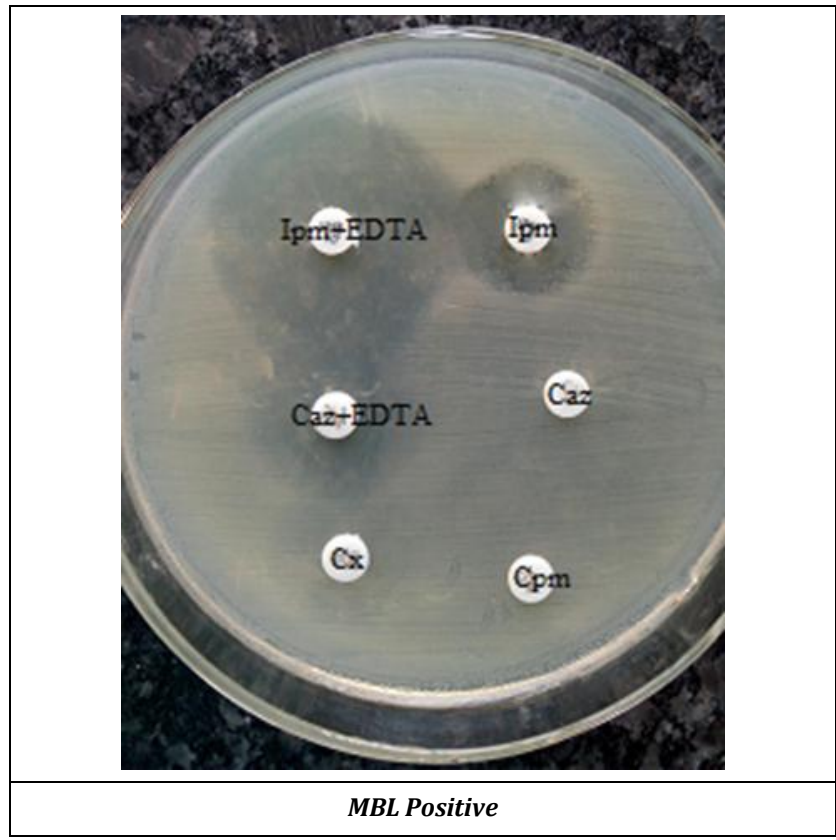

CAC- Ceftazidime + Clavulanic acid, Caz- Ceftazidime, CECCefotaxime + Clavulanic acid, Ctx- Cefotaxime, Cx- Cefoxitin, $\mathrm{Cx}+$ PBA- Cefoxitin + Phenylboronic acid, Imp- Imipenem, Imp + EDTA- Imipenem + EDTA.

\section{DISCUSSION}

The microbial flora of CSOM varies regionally, depending on the type of otitis media, use of antibiotics in that area etc. Nowadays, it is also common for an otolaryngologist to encounter bacterial flora of a chronic discharging ear that has already been modified by previous topical antibiotic therapy. Knowledge of the pathogen is therefore mandatory before initiating therapy in CSOM. The most commonly affected age group in the present study was between $36-55$ years. $61 \%$ of the affected patients were females and only $39 \%$ were males. This was consistent with the study by Loy AH et al. ${ }^{5}$ But most of the other studies have shown opposite trend with male preponderance. This is because males are usually considered 
to be more vulnerable to infection than females. The reason for this could be that the androgens in males and estrogens in females modulate host immunity, and these sex steroids affect disease resistance genes and behaviour. ${ }^{6} 2 \%$ had history of allergy. Because of the close anatomical relationship between eustachian tube and nasopharynx, allergic disorders like allergic rhinitis may cause eustachian tube dysfunction by inflammation and swelling in the area. ${ }^{7}$

Unilateral ear involvement (That too more on left side) in CSOM has been found more commonly than bilateral ear involvement. $49 \%$ of the cases had discharge from the left ear, $38 \%$ from the right ear and $13 \%$ from both ears. This was in concordance with study of Kumar N.et al. where $58.3 \%$ of the patients had pathology in the left ear. Pure culture was obtained from 98 samples, two samples yielded mixed bacterial growth and no bacterial growth was obtained in 35 samples. Negative culture can be attributed to: (1) Anaerobic growth (2) Prior treatment (3) Presence of antimicrobial enzyme. Out of the pure cultures, 66 isolates were Gram negative bacilli, 32 were Gram positive cocci and two contained both Gram positive cocci and Gram-negative bacilli. The predominant isolate obtained was Pseudomonas aeruginosa constituting 37\% (50 isolates) of the isolates followed by Staphylococcus aureus which contributed to $21.5 \%$ (29 isolates) of the total isolates. Many studies from different countries including India, Nepal, Nigeria and Singapore have reported that P. aeruginosa is the most common pathogen that caused CSOM, followed by S. aureus (Yeo et al. ${ }^{8}$, Madana et al.(32\%), ${ }^{9}$ Afolabi et al.(31.3\%). ${ }^{10,11}$ Whereas, studies from Pakistan, Iran and Saudi Arabia have shown that $\mathrm{S}$. aureus as the predominant pathogen, followed by P. aeruginosa (Mariam et al. (65.2\%). ${ }^{12}$ Possible explanation to this difference in isolation rate might be related to the effect of climate.

Pseudomonas survives competition with other pathogens due to its minimal nutritional requirements and production of antibacterial products like pyocyanin and bacteriocin. Klebsiella pneumoniae constituted $5.9 \%$ of the cases whereas among other Enterobacteriaceae, Escherichia coli contributed to $3.7 \%$ of the cases, Enterobacter aerogenes $1.5 \%$ and Proteus mirabilis $0.7 \%$. When the immunological resistance is low, organisms like Escherichia coli and Klebsiella species can become opportunistic pathogens in the middle ear. More frequent isolation of fecal bacteria like E. coli, Klebsiella and water bacteria like Pseudomonas indicates that the individuals are at a high-risk of infection due to poor hygienic conditions.

Ciprofloxacin (Quinolones) and gentamicin are usually given as topical antibiotics to patients diagnosed with CSOM. Quinolones are the preferred and most commonly used antibiotics due to their established effectiveness (Aslan et al.) and also because of them having lesser side effects (Cochleo toxicity and vestibulo toxicity). So, they are superior to aminoglycosides. ${ }^{13}$ In the present study, Pseudomonas aeruginosa showed only $36 \%$ susceptibility to Ciprofloxacin, $48 \%$ susceptibility to Gentamicin and $38 \%$ to the cephalosporin with anti-Pseudomonas activity, Ceftazidime. The other antipseudomonal cephalosporin, Cefepime though showed $54 \%$ susceptibility. The decreased susceptibility of Pseudomonas aeruginosa to the quinolone family is indicative of rapid appearance of antibiotic resistant strains. Quinolones are a critical group of antibiotics widely used in diverse infections like respiratory, urinary, bone and joint infections which could be the one of the reasons for the drug to develop resistance. The empirical treatment of patients with CSOM by quinolones without antibiotic susceptibility testing or culturing may reinforce resistance to quinolones. Parenteral antibiotics are given when the pathogen is found resistant to both the topical and oral antibiotics. The increasing resistance to parenteral antibiotics like the cephalosporin group is a warning sign, as these are potent antipseudomonal antibiotics. The most sensitive antibiotics in this study were Piperacillin-tazobactam, Imipenem and Amikacin. This finding was in agreement with the recent study which recommended the use of Piperacillin plus tazobactam like combinations against $P$. aeruginosa infections. ${ }^{14}$ Jang C.H et al. had similar findings in their study with Imipenem being the most susceptible antibiotic with an overall susceptibility rate of $96.5 \%$, followed by Amikacin (55.6\%), Piperacillin-tazobactam $(37.5 \%)$ and Ceftazidime (31.8\%).

Staphylococcus aureus was found to be susceptible to most of the antibiotics except Penicillin, to which only two out of the 29 isolates were susceptible. Among the 29 isolates of Staphylococcus aureus, all were Methicillin Sensitive Staphylococcus aureus (MSSA). This finding was contrary to the expectation in the current scenario that infections caused by MRSA would rapidly increase due to the misuse of antibiotics. Among the MDR Gram Negative isolates, organisms resistant to the Penicillins, first, second and third generation cephalosporins but not the cephamycins or carbapenems were tested for the presence of ESBL and AmpC. 20 isolates (50\%) among the total of 40 multidrug resistant isolates were found to be ESBL producers in this study, which contained 15 isolates of Pseudomonas aeruginosa, two isolates each of Klebsiella pneumoniae and Escherichia coli and one isolate of Proteus mirabilis. Study by Baguma A et al. had $89 \%$ of the bacteria being ESBL producers with the predominant isolate being Klebsiella pneumoniae (52\%), followed by Escherichia coli (44\%) and Proteus mirabilis (4.5\%). ${ }^{15}$ Most of the ESBL producing isolates were found to be resistant to Ciprofloxacin, Gentamicin along with Cefazolin, Ceftriaxone/ Ceftazidime and Cefepime. This was in concordance with study conducted by S.Shaikh et al. where the ESBL positive Pseudomonas aeruginosa showed 95\% resistance to Gentamicin, 91\% resistance to Ceftazidime, $85 \%$ resistance to Amikacin and $74 \%$ resistance to Ciprofloxacin and $65.96 \%$ resistance to Cefepime. It was $100 \%$ sensitive to Imipenem and Meropenem. ${ }^{16} \mathrm{High}$ percentage of ESBL producing isolates in the study could be due to selective pressure imposed by the frequent use of antibiotics.

In this study, 10 multidrug resistant isolates were $\mathrm{AmpC}$ producers. AmpC producers were also 100\% susceptible to Imipenem and Piperacillin-tazobactam. About $50 \%$ of the AmpC producers were susceptible to Ciprofloxacin and Gentamicin. 9 isolates of which were Pseudomonas aeruginosa and one isolate was Enterobacter aerogenes. This was in concordance with Wassef $\mathrm{M}$ et al, where out of the 50 isolates, 32 of AmpC producers were Pseudomonas aeruginosa, 10 were Klebsiella species, three Escherichia coli, three Enterobacter species and two Acinetobacter species. ${ }^{17}$ In a similar study conducted by Jameel NA et al. AmpC $\beta$ lactamases producing $E$. coli was found to be significantly 
resistant to Co-trimoxazole (91.8\%), Cefpodoxime (87.1\%), Aztreonam (69.4\%), Gentamicin (62.4\%), Amikacin (61.2\%) and Ciprofloxacin (34.1\%). They were found to be less resistant to Cefepime (7.1\%) and Piperacillin-tazobactam (5.9\%). ${ }^{18}$ High prevalence of AmpC beta lactamase producing Pseudomonas aeruginosa (22\%) has also been reported in a study done by Bhattacharjee A et al ${ }^{19}$ and $17 \%$ in a study conducted by Arora $\mathrm{S}$ et al. Therefore, compared to ESBL producers, isolates producing AmpC $\beta$-lactamase were resistant to additional $\beta$-lactams and to the currently available $\beta$-lactamase inhibitors. They also have the potential for developing resistance to carbapenems. Once the isolate was found to be resistant to carbapenems also, presence of carbapenemase was tested. 10 isolates of MBL producers were found in this study, majority being Pseudomonas aeruginosa (Seven isolates) followed by Klebsiella pneumoniae (two isolates) and Enterobacter aerogenes (One isolate). In a study on prevalence of MBL conducted by Jena J et al, out of the 112 Multi drug resistant strains, 20 isolates were found to be MBL producers. ${ }^{20}$ Maximum production was for Klebsiella oxytoca (75\%) and Citrobacter species (75\%). Resistance to newer drugs like Meropenem and Imipenem is a warning sign for the judicious use of carbapenems because Imipenem is regarded as the final medication for MDR Pseudomonas infection. Strains resistant to Ceftazidime, Cefepime and Imipenem are difficult to treat due to lack of antibiotics. Such cases require treatment with Polymyxin which is associated with severe nephrotoxicity and neurotoxicity. The high level of resistance seen in the isolates of P. aeruginosa could attribute to the fact that incomplete or partially treated cases are the ones mostly reaching tertiary care referral center. ${ }^{21}$

\section{CONCLUSIONS}

In the present study, high level of resistance to the commonly used antibiotics for CSOM like Ciprofloxacin and Gentamicin was observed. The second line drugs like Amikacin, Linezolid, Imipenem and Piperacillin-tazobactam were more effective. But as most of these drugs are intravenous antibiotics which are costlier and require hospital admission, it is difficult to start them for all the patients with uncomplicated CSOM. This situation can only be controlled by the judicious use of antibiotics. Since empirical therapy is often started for the patients attending the clinic with features of CSOM, it is prudent to do the antibiotic susceptibility testing regularly and be familiar with the susceptibility patterns in the hospital. Regular monitoring of the antibiogram will aid in the development of an antibiotic policy in the hospital which in turn will help in promoting rational use of antibiotics and prevent emergence of resistance. Also, testing for the $\beta$ lactamases are not routinely done in the laboratory. This may cause dissemination of ESBL producing strains within and between hospitals which may remain undetected for long periods.

\section{ACKNOWLEDGEMENT}

We are thankful to all the teaching and non-teaching staff of Department of Microbiology and Department of E.N.T, GMC Thrissur, for their valuable inputs.

\section{REFERENCES}

[1] Glasscock A, Shambaugh E. Surgery of the ear. 17th edn. Ontario: BC Decker Publisher 2006.

[2] Gupta G, Tak V, Mathur P. Detection of AmpC $\beta$ Lactamases in Gram-negative Bacteria. J Lab Physicians 2014;6(1):1-6.

[3] Kumar KGR, Navya S, Basavarajappa KG. A study of bacterial profile and antibiotic susceptibility pattern of chronic suppurative otitis media among patients attending a tertiary care centre, Davangere. Scholars Journal of Applied Medical Sciences 2014;2(5B):160612.

[4] CLSI. M100 Performance standards for antimicrobial susceptibility testing. 27th edn. 2016.

[5] Loy AH, Tan AL, Lu PK. Microbiology of chronic suppurative otitis media in Singapore. Singapore Medical Journal 2002;43(6):296-9.

[6] Klein SL. The effects of hormones on sex differences in infection: from genes to behavior. Neurosci Biobehav Rev 2000;24(6):627-38.

[7] Lack G, Caulfield H, Penagos M. The link between otitis media with effusion and allergy: a potential role for intranasal corticosteroids. Pediatr Allergy Immunol 2011;22(3):258-66.

[8] Yeo SG, Park DC, Hong SM, et al. Bacteriology of chronic suppurative otitis media-a multicenter study. Acta Otolaryngol 2007;127(10):1062-7.

[9] Madana J, Yolmo D, Kalaiarasi R, et al. Microbiological profile with antibiotic sensitivity pattern of cholesteatomatous chronic suppurative otitis media among children. Int J Pediatr Otorhinolaryngol 2011;75(9):1104-8.

[10] Afolabi OA, Salaudeen AG, Ologe FE, et al. Pattern of bacterial isolates in the middle ear discharge of patients with chronic suppurative otitis media in a tertiary hospital in North central Nigeria. Afr Health Sci 2012;12(3):362-7.

[11] Afolabi OA, Aremu SK, Alabi BS, et al. Traumatic tympanic membrane perforation: an aetiological profile. BMC Research Notes 2009;2:232.

[12] Mariam KA, Ahsanullah M, Mehtab J, et al. Prevalence of bacteria in chronic suppurative otitis media patients and their sensitivity patterns against various antibiotics in human population of Gilgit. Pak J Zool 2013;45(6):1647-53.

[13] Aslan A, Altuntas A, Titiz A, et al. A new dosage regimen for topical application of ciprofloxacin in the management of chronic suppurative otitis media. Otolaryngol Head Neck Surg 1998;118(6):883-5. 
[14] Javiya VA, Ghatak SB, Patel KR, et al. Antibiotic susceptibility patterns of Pseudomonas aeruginosa at a tertiary care hospital in Gujarat, India. Indian J Pharmacol 2008;40(5):230-4.

[15] Andrew B, Kagirita A, Bazira J. Prevalence of extendedspectrum beta-lactamases-producing microorganisms in patients admitted at KRRH, Southwestern Uganda. International Journal of Microbiology 2017;2017:3183076.

[16] Shaikh S, Fatima J, Shakil S, et al. Prevalence of multidrug resistant and extended spectrum betalactamase producing Pseudomonas aeruginosa in a tertiary care hospital. Saudi Journal of Biological Sciences 2015;22(1):62-4.

[17] Wassef M, Behiry I, Younan M, et al. Genotypic identification of AmpC beta lactamases production in Gram negative bacilli isolates. Jundishapur Journal of Microbiology 2014;7(1):e8556.
[18] Jameel NU, Ejaz H, Zafar A, et al. Multidrug resistant AmpC $\beta$-lactamase producing Escherichia coli isolated from a paediatric hospital. Pakistan Journal of Medical Sciences 2014;30(1):181-4.

[19] Bhattacharjee A, Anupurba S, Gaur A, et al. Prevalence of inducible AmpC $\beta$ - lactamase producing Pseudomonas aeruginosa in a tertiary care hospital in northern India. Indian J Med Microbiol 2008;26(1):8990 .

[20] Jena J, Debata NK, Subudhi E. Prevalence of extendedspectrum-beta-lactamase and metallo-beta-lactamase producing multi drug resistant Gram- negative bacteria from urinary isolates. Indian J Med Microbiol 2013;31(4):420-1.

[21] Kashyap S, Pandey A, Thakuria B, et al. Resistant microorganisms isolated from cases of chronic suppurative otitis media: a therapeutic concern. National Journal of Laboratory Medicine 2017;6(2):M001-M006. 